Rev. Latinoam. Psicopat. Fund., São Paulo, v. 12, n. 3, p. 469-480, setembro 2009

\title{
Adolescence et violence: une faillite de l'alterite*
}

Michèle Benhaim

A partir d'une clinique de l'acte adolescent dans son rapport au discours politico-social, seront évoqués la singularité du rapport à l'autre chez certains adolescents qui transgressent inlassablement les lois de la cité, comme s'ils étaient confrontés aux limites de ce qui ne fait pas, pour eux, expérience, d'autre part, le "pousse à l'acte" que recèlent certains pans du discours politique lorsqu'il est face aux débordements agis d'une partie de sa jeunesse, enfin, quelques repères relatifs au "traitement" de cette clinique contemporaine.

Mot clés: Acte, perversité, discours politique, adolescent, transfert

* Ce texte fut présenté oralement le Vendredi 28 Novembre 2008 au Laboratoire de Psychopathologie Fondamentale de l'Université de Campinas - Unicamp (Campinas, SP, Brésil) 


\section{Introduction}

L'adolescence, temps de franchissement qui voit le sujet tenter de se frayer une place dans le monde adulte, semble être un temps particulièrement propice aux passages à l'acte.

La violence qui peut caractériser ces passages à l'acte témoigne d'une impasse dans laquelle l'adolescent se débat.

Par ailleurs, elle s'accompagne presqu'inévitablement de marques de défaillances de la parole et du sens.

Quelques éléments de réflexions peuvent être dégagés de la clinique de certaines mises en actes adolescentes, qui à l'inverse d'actes véritables qui engagent, se révèlent être des agirs impulsifs et réitérés, et ce, chez de jeunes garçons rencontrés au sein d'une consultation qui s'inscrit au détour de leur placement judiciaire.

L'analyse d'une de ces rencontres cliniques révèlera la singularité du rapport à l'autre chez certains adolescents qui transgressent inlassablement les lois de la cité, comme s’ils étaient confrontés aux limites de ce qui ne fait pas, pour eux, expérience.

\section{Le cadre général de la consultation}

Les adolescents que je suis amenée à rencontrer ont fait l'objet d'un placement judiciaire suite à une accumulation de “délits”. Il apparait, dans ce processus de répétition, que la plupart de ces jeunes surenchérissent toujours plus loin dans la mise en acte de violences agies et/ou subies. Le processus s'emballe.

Les lois de la parole et du langage (syntaxe, règles de substitution d'un signifiant par un autre) reposent sur le passage de l'appel (le cri inarticulé du nourrisson) à la demande. Il semble que la 
déliquescence actuelle de ces lois (est "barbare” celui qui, étymologiquement, est tombé hors du langage) entraîne le constat que la division du sujet ne va plus de soi.

C'est ce qui produirait une sorte de catastrophe subjective que l'on pourrait définir ainsi: à l'aliénation symbolique d'un sujet, c'est-à-dire sa soumission, du fait qu'il parle, aux lois de la parole et du langage, ces signifiants thésaurisés dans l'Autre et dont il lui est fait don, à cette aliénation symbolique qui s'avère donc être structurante, va faire place, comme nous le montrera le cas de Paul, une aliénation sociale d'un individu captif d'un discours qui prône un rapport à l' objet immédiat, et qui le maintient ainsi dans le leurre que la satisfaction obtenue par la consommation de cet objet, serait totale.

Les adolescents parlent souvent de leurs passages à l'acte comme d'un temps où ils n'y étaient pas, pris soudain dans une sorte de vertige dû à un sentiment de vide et de solitude absolue ou d'envahissement un peu indéfini. Ainsi, en “panne” d'autre (Rassial, 1999), le sujet se structure mal et ce défaut de construction interroge le rapport à l' altérité qui en découle.

Ici se distingue la position que cette logique induit chez l'adolescent: la toutepuissance narcissique qui verse dans le déni de sa propre altérité, et, a fortiori, de celle de l'autre.

Si parfois c'est la mélancolie, ou au moins la dépression adolescente, trop intense, trop longue pour n'être que du registre de la "morosité" due aux deuils en cours d'accomplissement, si c'est la mélancolie donc qui se manifeste, c'est parce que le sujet s'effondre face au constat d'échec auquel mène, finalement, cette logique. C'est pourquoi le diagnostic de “bi-polarité”, reposant sur l'alternance d'une clinique de la violence adolescente assortie de passages à l'acte et de troubles dépressifs accompagnés de repli sur soi et/ou de tentatives de suicide, est de plus en plus fréquemment posé sur de jeunes sujets.

Avançons que cette "bi-polarité” reflète pour une part celle de son inscription dans le discours qui le parle, comme une réponse en miroir possible face aux alternances des injonctions sociales où tantôt il lui est promis un bonheur illimité, tantôt des réponses toutes répressives viennent (trop) brutalement rappeler le principe de réalité au souvenir de l'adolescent.

La violence que certains adolescents mettent à l'ceuvre sans frein témoigne d'une logique que l'adolescent dénonce et partage tout à la fois.

Il a "la haine” de celui là même dont il emprunte la logique subjective. La “bi-polarité” du social tour à tour “pervers” et mélancolique (Douville, 2000) ferait il le lit des spectaculaires dépressions adolescentes sur le premier versant, et des hyper-agitations du jeune, sur le second?

L'adolescent ne peut, dans la plupart des cas, nommer ce sentiment de chute vertigineuse ou d'impulsiôn irrépressible qui surgit à son insu (scarifications, 
TS...). Comme s’il était captif d'une mauvaise rencontre qui ne peut se symboliser, l'adolescent fera appel, non pas via le symptôme mais à travers le passage à l'acte, acte répétitif parce que le plus souvent sourd à l'appel.

A l'appel, un autre se doit d'être à l'origine d'une réponse dont l'objectif visera à signifier au sujet en train de se constituer qu'il va falloir parler pour demander, désirer pour être, témoigner ainsi, en quelque sorte, d'une bonne intégration de la perte fondamentale de l'objet.

Les défaillances symboliques du monde adulte aujourd'hui privent d'appui ces adolescents.

Si l'aménagement familial repose sur une logique pervertie par une mère qui jouit de l'enfant avec la complicité d'un père qui assiste au "caprice” sans le tempérer, voire qui y participe (on pense ici aux situations incestueuses ou de maltraitance mais d'autres situations, moins bruyantes, relèvent de cette même logique), nulle instance rie pourra s’instaurer sur le versant d'une autorité. L'enfant invalidera sur la scène familiale et/ou scolaire des éléments de ce fonctionnement pathogène en le dénonçant et en s’y inscrivant en même temps.

Tout se passe comme si le(s) parent(s) ne signifie(nt) pas à l'enfant la dimension du manque. L'enfant devenu adolescent, c'est-à-dire bouleversé par l'exigence des vrais renoncements qu'exige le passage pubertaire (Gutton, 1991), les récusera comme Autre (parental) au lieu de grandir en acceptant de se confronter à ces “nouvelles” figures, nouvelles d'être à présent descendues de leur piédestal.

C'est ce processus que semblent recouvrir, à l'adolescence, les manifestations toute-pulsionnelles comme seule voie possible vers l'autonomie.

C'est alors la structure même de la parole qui fait problème et trouve à se résoudre dans le passage à l'acte.

Mais comment l'adolescent peut-il interpeller l'autorité adulte qui est censé le garantir dans l'existence et qui manque à sa place de restricteur de jouissance en se faisant, au contraire le modèle même de l'accomplissement de cette dernière? Ce que le sujet ne peut dire, ille met en scène, et il ne veut rien en savoir.

\section{Clinique}

Nous prendrons pour exemple ce couple de toxicomanes totalement inséré socialement et économiquement mais dont le lien faisant office de lien amoureux, consiste à "se défoncer" ensemble, c'est à dire à jouir, ensemble, mais seuls, et ce, sous le regard de leur fils depuis son plus jeune âge. Après une enfance traversée de la nécessité pour ce fils que nous nommerons Paul, de devoir prendre soin de ses parents, devenu adolescent, (et c'est le motif du placement et donc 
de la rencontre avec le couple) il va témoigner à travers toute une série de passages à l'acte à répétition (cambriolages) qu'il s'est résigné à s'inclure dans cette logique perverse. Je le reçois là où il me dit “ne pas comprendre ce qu'il fait là”. Récusant toute portée symbolique à son acte, il ignore "pourquoi on fait un drame" de cambriolages où les objets dérobés ont la particularité de ne servir à rien (inutilisables, invendables).

Voici donc un contexte où le processus adolescent s'exprime dans des mises en danger de l'autre ou de soi-même. Les repères symboliques ne font pas nécessairement défaut à ce jeune, loin d'être psychotique, et pourtant la fonction paternelle témoigne, au travers de l'anéantissement de toutes limites, de son inefficace opération.

Ce type de logique peut laisser des adolescents en butte à un nombre plus que réduit de points d'appui signifiants. Sans le recours possible aux repères fondamentaux, le réel pubertaire, familial et social, rencontré à cette occurrence de la vie qui signe le passage adolescent, reste insubjectivable et fait retour tel quel, dans l'acte. Ce qu'il manifeste doit lui rester étranger. Paul ne reconnait pas la portée de ce qu'il montre. Il “se débrouille” de cette invitation parentale à un échange de type pervers, d’une économie familiale qui opère dans un désarrimage du désir sans limite et de la loi.

Ce qui supplée ici à la perte inexorable mais nécessaire de l’objet, ce n’est pas l'amour, c'est l'injection partagée. Autrement dit, un vecteur de transmission actuel réduit à un désir maternel illimité partagé par le couple parental.

Pour ce fils à la mère excessive pour ne pas dire "abusive”, rien ne fait point d'accroche aux discours éducatifs qui se veulent pourtant structurants mais qui se figent face à l'énigme sans cesse répétée de cette accumulation de passages à l'acte que le placement n'a pas interrompus, et que rien ne semble pouvoir être à la hauteur d'accueillir dans une possible élaboration symbolique. L'autre est seulement mobilisé comme un regard, dans la seule dimension imaginaire. La dimension symbolique qui permettrait de décoder ce qui est montré ainsi, se trouve exclue dans cette forme d'impasse.

Le processus semble sans fin. Ce qui fait d'un in-dividu un sujet, c'est d'être sujet d'expérience et d'historicité.

Cet adolescent "ne voit pas où est le problème”: il semble agir sans pouvoir penser, errer au fil d'une absence de rêverie là où le socio-éducatif lui fait injonction de construire un projet, être dans la désertion de son monde psychique interne là où on lui demande de s’inscrire dans une dimension sinon collective, du moins institutionnelle. Pour tirer leçon de l'expérience, encore faut-il être affecté. De plus, la subjectivité ne peut se mettre en jeu, à l'adolescence justement, que dans l'articulation à d’autres. Ici les épreuves s'accumulent sans être éprouvées

Rev. Latinoam. Psicopat. Fund., São Paulo, v. 12, n. 3, p. 469-480, setembro 2009 
et, sur ce passage d’épreuve impossible, repose le processus de désubjectivation profonde.

Il n’a “même pas mal”, comme s’il évoluait par à-coups, sans temps ni espace pour l'ébauche d'une construction de symptôme. Sans une historicité qui noue l'expérience à l'altérité, on ne construit pas son histoire à partir d'expériences avec d'autres et on demeure sujets de “rien”, c'est à dire individus.

Paul fugue dès que les éducateurs ne le regardent plus et commet des vols qui le mettent de plus en plus en danger, s’enfermant dans une sorte d'impasse associée à toutes les formes de déviance perverse dont il nous fait les témoins. (Il accumule en effet les transgressions: fait fumer du cannabis aux plus jeunes, conduit sans permis, tente de contraindre quelques filles à des relations sexuelles qu'elles ne souhaitent pas, agresse les éducatrices.)

Ces enjeux violents témoignent de la perte d'un espace psychique qui appartient au sujet, sensé faire axe, représenter un appui de référence qui permet la rencontre.

Ses mises en scène correspondent à une forme particulière de souffrance liée à la défaillance de la structure symbolique de ceux qui étaient censés exercer une responsabilité à son égard.

Paul, dans une illusoire existence, dénuée, croit-il, d’angoisse, évolue dans un rêve de suffisance narcissique d'où l'autre, et la génitalité qu'il engage, sont absents.

Cette impossible construction semble prendre source dans un défaut de lien antérieur où les seules identifications se sont effectuées sur le modèle de figures perverses, modèle ne pouvant donner lieu qu'à cette sorte d'espoir d'auto-engendrement où la satisfaction s'obtient seul.

L'addiction, reprise aujourd'hui à son propre compte, aune fonction autocalmante comme si c'était un organisme qu'il fallait faire jouir, et non un corps qu’il fallait faire circuler dans une économie relationnelle désirante. Comme si était effacé, au départ, l'effet de l'amour qui aurait pu l'introduire à une dimension érotique.

Le passage à l'acte n'apaise pas Paul, il l'épuise; il ne lui fait pas non plus horreur, il l'indiffère.

Que nous révèle l'isolement de Paul qui n'a rien à dire?

Dans quelle dimension s'est inscrit l'Autre?

Il y a absence de prise en compte de l'altérité. Ses tentatives de violences sexuelles s'inscrivent dans un effacement de l'altérité. Il n’y a pas de place pour deux: l'un évince l'autre, le tue, le viole, l'annihile. Comme une façon pour lui de dénoncer cette absence vécue, cette place occupée d’objet à consommer, et de pointer le leurre d'une rencontre ou d'un dialogue autres que pervers. Paul voit 
ses passages à l'acte se tordre et s'emballer: il devient, en quelque sorte, socialement fou.

Dans ses mises en actes, cet adolescent ne souffre pas, ne se plaint pas, ne témoigne en rien de ce qu'il donne à voir pourrait le toucher. C'est le témoin qui souffre. Il va s'agir d'entendre ce qui est donné à voir sans céder à l'évidence de la portée symptomatique de l'acte.

Si on considère bien cette fausse évidence, autrement dit que l'adolescent agit non pas parce qu'il est dans une incapacité à dire mais parce qu'il est confronté à une impossible parole, on peut s'attendre à ce qu'un transfert s'établisse et offre la condition d'une référence symbolique qui permette un travail psychique en réintroduisant la possibilité de parler.

D'ailleurs, peu à peu, un discours chez cet adolescent s'esquisse: d'une part la répétition et l'escalade de ses mises en acte commence à lui faire question, et, d'autre part, il commence à mettre en récit des morceaux épars de son roman familial. Ce mouvement nous indique que ses dénis étaient bien et paradoxalement une adresse à l'autre et que nous avions raison d'en tenir le djfficile pari. Il aimerait que la menace d'emprisonnement qui pèse sur lui fasse au moins rupture dans la consommation de drogues effrénée de ses parents et surtout de sa mère.

L'acte met en scène le sujet dans un rapport à l'objet auquel il a accès, lui aussi. Les objets volés, ceux qui ne servent à rien, se rapportent pour l'essentiel, à du matériel médical: est-ce le symbolique familial et social qui est ici détruit par l'acte?

La consommation parentale excessive, parce qu'exhibitionniste, est perverse et parallèlement, la demande pervertie faite à l'adolescent, celle en l'occurrence de prendre soin de ses parents, soin dont on soupçonne qu'il consistait, entre autre, à leur acheter du matériel d'injection, cette demande met à l'écart sa subjectivité qui fait retour dans le réel du passage à l'acte.

Dans une séparation impossible d'avec sa mère, l’objet s’est toujours présenté sur fond de présence.

Voici un autre exemple clinique.

David fume du cannabis à longueur de temps: “je fais le vide”, me dit-il. C’est dans ce contexte de court-circuit émotionnel et affectif que le désir semble s’effacer. Les éducateurs se plaignent beaucoup de lui, il ne parvient pas à tenir la moindre activité, encore moins le moindre projet éducatif. David explique les délits qui l' ont conduit au foyer (braquages de stations service en bande) ainsi: “je ne peux pas m'empêcher d'y aller, voir la tête des mecs qui tiennent la caisse quand on empile les packs de bière, c' est ça que j' aime...”. Dans un emballement pulsionnel inquiétant, le sujet entretient un lien aliénant non pas à l' autre mais à son presque symptôme: c'est lui et lui. 
En face de David, le discours social de l'insertion, mais David ne produit ni ne reproduit: il passe à l'acte.

Cet élément est directement et intensément à l'oeuvre dans la sexualité à laquelle David et sa bande s’essayent et qui représente un vrai danger pour ces adolescents qui vont mal et qui se font mal. Ils y réalisent une sexualité non génitale, hors responsabilité, c'est à dire hors rencontre de l'autre. Soit le partenaire n'est pas appréhendé comme un sujet différencié ayant son propre désir mais comme pur objet de consommation sexuel, soit ils s’y jettent sans distance aucune, livré sans médiation à la demande impérative de l'Autre. L'adolescent comme l'autre sont devenus des êtres-objets à valeur exclusive d'usage.

Lacan (1986) soulignait comment le filtre de la famille pouvait se faire poreux et "laisser l'enfant ouvert à toutes les prises fantasmatiques". Dans les cas rencontrés aujourd'hui, la famille, on l'a vu chez Paul, se fait parfois complice et laisse l'enfant aux prises avec une essence de la perversion, éléments que les adolescents rapportent souvent comme ils raconteraient le film télévisé de la veille; Pour que le sujet participe de ce qui lui arrive, encore faut-il qu'il puisse penser qu'il lui arrive quelque chose, or, nous l'avons dit, la répétition ne fait pas sens, l'expérience ne se déduit pas comme telle et les sujets “abandonnés” font de l'abandon lui même, une jouissance.

Un échec de la symbolisation et du refoulement, un défaut de fantasmatisation qui peut se traduire par des agirs pathologiques pulsionnels, ce pulsionnel qui ne trouve pas d'autre voie de décharge, ce défaut d'ouverture à l'altérité, cette rencontre devenue impossible, ce temps où la mort est déliée de la culpabilité.

Tout adolescent doit répondre d'une angoisse existentielle qui signe le passage de l'infantile au juvénile. Il peut en passer par des “agirs” pour échapper à l'emprise angoissante du désir de l'Autre. Mais, il arrive qu'ils rencontrent des écueils qui les empêchent de construire un lien social, un symptôme. C'est ce dont témoignent les mises en acte de la limite et son franchissement dans la violence ou dans la mort. Ces actes sont comme des ultimes tentatives de créer le signifiant qui les représenterait auprès des autres.

Ils semblent alors faire preuve d'un narcissisme illimité bien que défaillant et passent à l'acte dans le souci de créer une coupure qui les départirait de l' envahissement de l' objet. Cependant, l'autre face de ce mouvement pathologique nous autorise à faire le pari que cet acte continue d'être adressé. En effet, lorsque c'est l'autre même qui se trouve être l'objet de cette violence, il y a comme une tentative d'en finir avec son injonction.

Paul agresse les éducatrices. Il "cogne” celles là même qui le nomment à une place où il disparait, agissant ainsi ce que justement il tente d’ invalider. 
L'adolescent se détennine ici dans son acte et non dans une nomination. Ou plutôt dans une nomination qui lui échappe. Des signifiants tels que “délinquant” l'assignent mais ignorent - son être.

\section{Conclusion}

Nous conclurons sur quelques réflexions quant au traitement clinique de ces psychopathologies dont la difficulté relève justement de la dimension perverse qu'elles contiennent.

Identifié à son acte, l'adolescent est peu enclin à la parole, à la demande, au transfert, ainsi, alors même que les conditions propices au transfert sont absentes, il nous faut “en prendre l'initiative” en engageant notre parole, c'est à dire notre division propre, ce qui fait justement question chez l'adolescent. "To meet”, dirait Winnicott (1971), aller rencontrer l'adolescent comme moyen pour ce dernier “d'enfin rencontrer l'autre”. Cette rencontre est nécessaire pour pennettre au sujet de découvrir "son self propre et une relation au monde telle qu'il pourrait la ressentir comme réelle".

Dans un contexte pulsionnel où les mises en acte se succèdent sans donner lieu à des associations psychiques et verbales, il va nous falloir viser à réintriquer ce qui est donné en pâture au seul regard, à la parole. C'est à dire en passer par l'articulation d'une demande. Mais comment et pourquoi demander à un autre qui ne répond pas?

La position clinique n'a pas le choix que d'entendre un discours qui s’adresse, sinon, nous restons figés à notre tour dans l' angoisse que la répétition de ces actes produit et pouvons alors nous montrer défaillants.

La parole est récusée, aussi le clinicien parle pour signifier la contrainte de perte propre au langage: c' est ce qui s'oppose à leur silence et fait de l'espace thérapeutique un temps de rencontre, de réhumanisation.

La position clinique consiste ici en une sorte de "forçage" symbolique (pour reprendre un terme de Berg ès évoquant le lien de la mère à son bébé) qui ferait valoir une contradiction: bien sûr cela entraine des violences difficiles à travailler dans un premier temps pour que puisse se nouer le transfert. Ce forçage fera pourtant valoir la dimension d'appel; on va apprendre à l'adolescent à “parler”.

Au seuil de la parole, au seuil du symptôme, le travail clinique consiste à cicatriser ce vide du déni c’est à dire réintroduire la dimension de la perte en créant les conditions d'une énonciation. Le passage à l'acte nous situe en-deçà du langage, aussi nous faut-il engager l'adolescent à construire un récit qui borderait ces excès, à tisser une "fiction" dont se structurerait le symptôme comme un langage, comme un compromis. Introduire le temps et exiger la mise en récit, tel peut 
être le projet d’historicisation en face de l’im-médiateté prônée par le discours ambiant auquel nombre d'adolescents "collent”.

Le symptôme se structurera si la souffrance du sujet vient le nouer, aussi ne va t-on pas réparer mais réintroduire une temporalité, exiger le temps du récit, chez cet adolescent anesthésié par la promesse de jouissance qui le leurre sur ses propres ressentis, construire un après-coup, nommer un point d'insupportable, en l'occurrence ce point dans le symptôme qui pourrait amener le sujet à venir questionner, voire à venir demander à (ré)inventer sa place dans le monde.

Cette clinique des actes adolescents sollicite le clinicien dans son identité même, dans sa responsabilité et dans son éthique.

Voici l'exemple d'une histoire adolescente récente qui montre combien les défauts de structuration psychiques de certains adolescents se trouvent traversés par les symptômes de notre société: je reçois un peu dans l'urgence, la bellemère de Marie, 16 ans, (la femme de son père). Celle-ci est très angoissée et me fait part de l'hospitalisation de Marie suite à une tentative de suicide. La jeune fille va bien, aussi ce n'est pas la TS qui met la belle mère dans cet émoi mais les conditions de cette TS: en effet, à l'arrivée des pompiers appelés par le père (la mère de Marie, quant à elle, avait eu comme première réaction de téléphoner au petit copain de sa fille et de l'injurier copieusement, prétendant qu'il était responsable de cette TS dans la mesure où il avait fait part à Marie d'une rupture), la belle mère constate horrifiée que la web cam de l'ordinateur est allumée et que Marie s'est tailladée les veines en live sur le net...

Marie semble avoir été soumise à l'impératif d’un spectacle: impératif véhiculé par le regard, stigmatisé et diffusé sans limite. Une certaine logique du regard et de l'image semble s'être ici substituée à l'organisation d'un lien. N'est-ce pas la conséquence de la logique du discours qui concerne les adolescents et de la place qui leur est ménagée par les adultes?

\section{References}

Douville, O. Pour introduire l'idée d'une mélancolisation du lien social. Cliniques méditerranéennes, Toulouse, n. 63, p. 239-262, 2000.

GutTon, PH. Le pubertaire. Paris: PUF, 1991.

Lacan, J. Deux notes sur l'enfant. Ornicar?, Paris, n. 37, 1986.

Rassial, J.-J. Le sujet en état limite. Paris: Denoël, 1999.

WinnicotT, D. W. Jeu et réalité. Paris: Gallimard, 1971. 
Resumo

(Adolescência e violência: uma falha da alteridade)

A partir da clínica do ato adolescente ligado ao discurso político-social, será analisada a singularidade da relação com o outro em alguns adolescentes que, incansavelmente, transgridem as leis da periferia, como se fossem confrontados aos limites daquilo que, para eles, não vale como experiência. Por outro lado, será também analisado "a passagem ao ato" que ocultam alguns segmentos do discurso político quando confrontado com ímpetos que excedem uma parte de sua juventude. Finalmente, serão abordados alguns pontos de referência para o "tratamento" ligado a essa clínica contemporânea.

Palavras-chave: Ato, perversidade, discurso político, adolescência, transferência

(Adolescencia y violencia: una falla de la alteridad)

A partir de la clínica del acto adolescente ligada al discurso político-social, será evocada la singularidad de la relación con el otro en algunos adolescentes que incansablemente transgreden las leyes de la periferia, como si fueran confrontados a los limites de lo que no vale, para ellos, como experiencia. Por otro lado, será también analizado el "pasaje al acto" que ocultan algunos segmentos del discurso político cuando confrontado con ímpetus que exceden una parte de su juventud. Finalmente serán abordados algunos puntos de referencia para el "tratamiento" ligado a esa clínica contemporánea.

Palabras claves: Acto, perversidad, discurso político, juventud, transferencia

(Adolescence and violence: a failure of otherness)

This article is based on clinical experience with adolescents and with political and social discourse. The authors analyze the singular relationship with the other that one sees in certain adolescents who constantly violate the laws of the barrio, as if they were confronted with the limits of what they refuse to accept as experience. "Passage to the act" is also analyzed, to the extent that certain segments of political discourse are concealed when confronted with impulses that adolescents seem unable to control. Finally, several points of reference for treatment in this type of contemporary clinical situation are discussed.

Key words: Acting, perversity, political discourse, adolescence, transferrence

Citação/Citation: Benhaim, M. Adolescence et violence: une faillite de l'alterite. Revista Latinoamericana de Psicopatologia Fundamental, São Paulo, v. 12, n. 3, p. 469-480, set. 2009.

Editor do artigo/Editor: Prof. Dr. Manoel Tosta Berlinck 
Recebido/Received: 19.12.2008 / 12.19.2008 Aceito/Accepted: 4.3.2009 / 3.4.2009

Copyright: ( 2009 Associação Universitária de Pesquisa em Psicopatologia Fundamental/ University Association for Research in Fundamental Psychopathology. Este é um artigo de livre acesso, que permite uso irrestrito, distribuição e reprodução em qualquer meio, desde que o autor e a fonte sejam citados/This is an open-access article, which permits unrestricted use, distribution, and reproduction in any medium, provided the original author and source are credited.

Financiamento/Funding: $\mathrm{O}$ autor declara não ter sido financiado ou apoiado/The author has no support or funding to report.

Conflito de interesses: $\mathrm{O}$ autor declara que não há conflito de interesses/The author declares that has no conflict of interest.

\section{MichèLe Benhaim}

Maître de Conférences Habilitée a Diriger des recherches en psychopathologie clinique et psychanalyse, Université de Provence Aix-Marseille, Laboratoire de Psychanalyse et de Psychopathologie Clinique.

8 Rue Alfred de Musset 23006 Marseille, France

e-mail: michelebenhaim@voila.fr 